\title{
Exploring Tone Variations in Chinese Dialects Using Context Dependent Tone Models
}

\author{
Wei Guo \\ Shanghai Jiao Tong University \\ Email:w_guo@sjtu.edu.cn
}

\author{
Min Chu \\ Microsoft Research Asia \\ Email: minchu@microsoft.com
}

\begin{abstract}
In this paper we propose a statistical approach for prosody study. It has two key stages: first, context dependent models are trained automatically from a natural speech corpus. Then data mining and data visualization techniques are used to discover phonetic knowledge from the model parameters. We use this approach to study the tonal system of Xi'an dialect, where we learn much knowledge about the dialect, without the need of any specific phonetic annotations about it. Some of our observations coincide with those from other studies, which demonstrate the capability of this approach, while some of our new findings show its advantages comparing with traditional methods.

Index Terms - tone variations, Chinese dialects, context dependent tone models
\end{abstract}

\section{INTRODUCTION}

Chinese has a large number of dialects. Although they share a similar writing system, these dialects have rather distinct phonetic systems. The same written word can be pronounced tonally different in different dialects. Studying the tonal system of a dialect and comparing tones across dialects are among the most important research areas in Chinese phonetic study. In traditional phonetic studies, tones in dialects are usually noted by experts, who have rich experiences in phonetics and are familiar with the target dialect, according to their perceptions. With their phonetic knowledge, these experts are able to identify the number of tones used in a certain dialect and the pitch patterns of these tones. In some studies, experts can also summarize rules to capture tone variations in simple contexts. Much knowledge about certain Chinese dialects, such as Cantonese, has been gathered through such traditional approaches. Yet, there are still many dialects waiting to be explored. There are two main drawbacks of the traditional approach that block the progress. First, accumulating knowledge on a certain dialect is a very time consuming process. Second, only a few experienced experts are capable of studying such topics. As a result, most studies of dialect tones focus more on the simple facts such as the pitch patterns of tones and the tone sandhi phenomena. The tonal variations in natural speech are seldom studied due to the complexity of handling multiple contextual factors together. Therefore, a more efficient way to explore the tonal systems of dialects is desperately in need.

In this paper, we propose a statistical paradigm for prosody or phonetic study of a language or a dialect that we don't have much knowledge about. First, statistical learning technologies are used to construct context dependent models (CDMs) automatically from a large scale speech corpus. Then, the statistics of acoustic features embedded in the models are used as the data source for prosody study. Finally, data mining and data visualization techniques are used to discover phonetic knowledge from the models. Such a statistical approach enables us to draw conclusions by exploring natural speech corpus in a large scale, which is very difficult to achieve with traditional ways. Furthermore, the context dependent models contain most of the information phonetists need for their study. Therefore less time should be spent on speech data collecting and processing. Instead, phonetists can examine the statistics of CDMs from various view points and learn rules or patterns from them. In this paper, we demonstrate the scenario by studying the tonal system of Xi' an dialect using recorded speech of a Xi' an resident.

\section{THE STATISTICAL PARADIGM FOR PHONETIC AND PROSODY STUDY}

In this paper, we choose Xi'an dialect as our research subject to prove the correctness of the statistical paradigm. Context dependent tone models (CDTM) in the form of MSDHMM (Multi-space Distribution Hidden Markov Models) [1] have been proved to have the capability of capturing the tone variations in different contexts in Mandarin [2][3]. They are used in this work to model the tone variations in the dialect. The challenges we faced are that we don't have much knowledge about Xi'an dialect except that it is related to Mandarin. Therefore, we use Mandarin phonetic notations, i.e. Hanyu Pinyin, to annotate the dialect speech for the start of our work. We hope that we can discover its distinct characteristics of the dialect in the process.

Since our focus is on the tonal system of the dialect. In this work, four contextual factors, including current tone (CT), left tone (LT), right tone (RT) and syllable position (SP) are considered. Each distinct context is modeled by a MSD-HMM, which is aligned to syllables in the speech. A four-state, leftto-right model structure is adopted. We expect the first state to capture the unstable portion in the $f_{0}$ curves of a tone. Most syllables begin with unvoiced initials whose corresponding speech segments quite often don't have $f_{0}$ values. But MSDHMM provides a multi-space representation of the $f_{0}$ feature, which saves us from handling the voiced/unvoiced cases specifically. In our case, one state in the model is already able to capture the initial portion. Then the rest 3 states are expected to represent the beginning, middle and end portion 


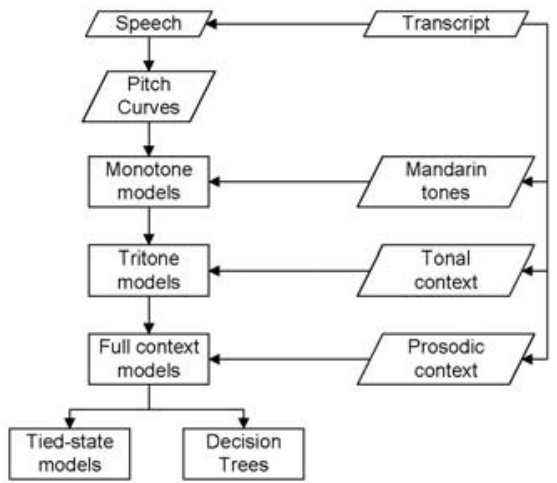

Fig. 1. Work flow of the model training process.

of tones and be able to capture the up-down or down-up type of pitch movements. The feature distribution within each state is described by a Gaussian model.

The CDTM training process is rather similar to that in [1] for Mandarin. The key difference is that only tone and position factors are considered in this work. The reason is that from the factor ranking in Mandarin, tone and position factors are the most important ones. Another difference is, in [2], CDTMs are trained for syllable initial and final respectively. In this work, CDTMs are trained for syllables as a whole.

As shown in Fig. 1, incremental training technique is used to ameliorate the impact of insufficient data. First, monotone models are trained using all the data. Then, monotone models are expanded to tritone models. Finally, tritone models are expanded by adding syllable position information. After having these tone models that reflect the tone pattern in specific contexts, we'd like to know which contextual factor has the most significant impact on tone patterns. Therefore, we use CART (Classification And Regression Tree) to cluster the models and consider the tree structure an indicator of the contextual impacts. We believe that different parts of a tone pattern may be influenced by different contextual factors, so we cluster these parts separately, i.e., states at the same position of all models are tied within the same tree. Questions regarding to all the four contextual factors are provided for splitting nodes. The tree is grown greedily by always splitting a node with a question that results in the largest likelihood increasing [4]. In this study, we apply the MDL (Minimum Discrimination Length) criterion for split stopping [5]. As a result, states of models with similar contexts are grouped together. We can also rank the importance of contextual factors by observing the trees, because questions used for early splitting have larger impact than those used for later splitting.

\section{EXPERIMENT AND RESULTS}

\section{A. Experiment setup}

We designed a reading script for dialect speech collection with our knowledge about Mandarin phonetic system. The script consists of 80 sentences, 3 poems, 2 movie monologues,

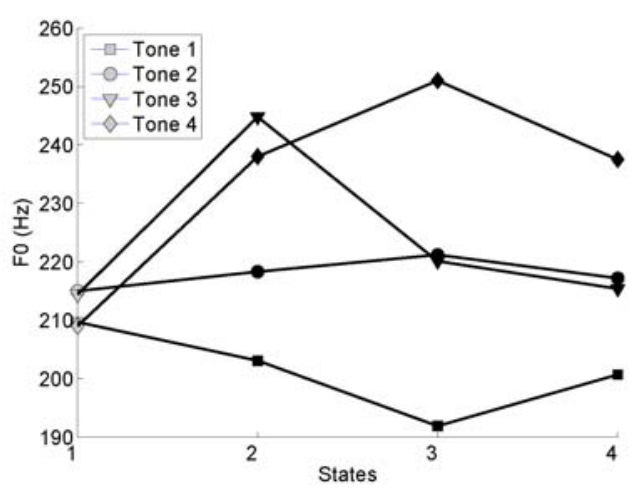

Fig. 2. Stylized $f_{0}$ patterns of mono tones for Xi'an dialect. Gray scale of the markers indicate the probability of the state been voiced.

and 2 news reports. 1400 syllables in total. A native Xi'an female speaker reads the script with the Xi' an dialect. Both her speech and EGG (Electroglottography) signal are recorded. $f_{0}$ curves are extracted from EGG signal, from which a five-dimensional feature vector are derived, which includes logarithmic $f_{0}$, its first and second order derivatives, 10 frame average, and long-span average [6]. Syllable boundaries are manually labeled. These boundaries are used in model training to align tone models to syllables.

In Mandarin, tone 1-4 represent the high-level tone, the rising tone, the dipping tone, and the falling tone, respectively. In the current study, they are symbols related to tones in the dialect. The syllable position has the same definition as in [2], which represents the two layer prosodic structure: prosodic word and prosodic phrase.

\section{B. Monotone pattern}

We first examine the monotone models. The $f_{0}$ mean of each state in the four tone models is plotted in Fig. 2. We use the darkness of the markers to show the probability of the state being voiced. Since the first state describes the initial portion of syllables and that portion is quite often an unvoiced phone, the first states for all the four tones mostly have a light shade and their $f_{0}$ values are closed to each other. State 2 to 4 reflect the pitch patterns of the four tones. It is very clear that the four tones have rather distinctive patterns. Therefore, we can conclude that $\mathrm{Xi}$ 'an dialect has at least 4 distinct tones. This is consistent with Li's description [7]. Furthermore, Li noted tone 1 to 4 as 21, 24, 53 and 44, respectively, using Yuanren Zhao's system of tone notations. From Fig. 2, we find that the movements from state 2 to state 3 for tone 1 , tone 3 and tone 4 match Li's notation well. But tone 2 shows as a rather flat middle level tone rather than a rising tone. The fourth state of each model captures the transition part from the current tone to the next tone. Generally, for a high-ending tone, the final part tends to move downward; for a low-ending tone, it tends to move upward; and for a middle-ending tone, it tends to keep flat. Since monotone models are intensively averaged across various contexts, the impact of contextual factors will not be 
seen unless we extend them to context dependent models. In section 3.4, We explores the tone variations in different context. Before that we first compare the impact of contextual factors on the four tones through analyzing the structure of the decision trees.

\section{Impact of contextual factors}

Fig. 3 shows the first 7 splits of the decision tree for tying the second states (which describe to the beginning portion of a tone) of all CDTMs. We find that the first and second questions separate tone 2 and tone 1 from the others which shows that start point of tone 1 and tone 2 are distinct from the other two tones. All other questions are about the left tonal contexts of the syllable, except for when the current tone is tone 1 . This shows that the beginning portion of a tone is mainly affected by the tone before it. But tone 1 is an exception because it has a tone sandhi phenomenon, which we will further investigate by visualizing these models in the next section. The impact of contextual factors can also be measured in a numerical fashion. From the tree growing procedure, we know that the earlier a question is used, the more impact it has on the state. We measure the impact of a question by the reciprocal of the depth of the node it splits, and the overall impact of a contextual factor is described by the sum of its impact measures from all questions regarding to it. Fig. 4 shows the impacts of the four contextual factors on the four states in CDTMs. As we can see, overall the current tone has the largest impact on tone models. The left neighboring tone also has a strong impact and its impact decrease from left to right. While the right neighboring tone shows an increasing impact on the tone models, especially on the latter two states. Although syllable position is considered in our study, it shows almost no impact on tone models. This observation is rather different from what is reported for Mandarin [2], where syllable position ranks on top. A possible reason is that the Mandarin speech corpus is read a broadcast woman who has very good control of the speech prosody, but, the dialect speech is read by a college student who hasn't paid much attention on speech prosody.

\section{Visualizing and exploring the tone variation in different context}

Beside ranking the impact of different contextual factors, we can also draw the stylized pitch patterns for tones in different context as shown in Fig. 5. Since syllable position shows no impact on tones in our data, we plot the four tones against the tones before and after them. From the left-most column in Fig. 5, we can see clearly a tone sandhi phenomenon, i.e. when a tone 1 (which is a low tone), is followed by another tone 1 , it will change to a rising tone, which is very similar to tone 2. Such a tone sandhi rule was also reported in [7]. Furthermore, we find that when a tone 1 is preceded by another tone 1 (which has changed to a rising tone) or is followed by a high-starting tone like tone 3 , it tends to reach a much lower beginning pitch than in other cases. Tone 2 shows two distinct patterns. When a tone 2 is preceded by another tone 2 , it tends to be a middle flat tone. In other cases, it is a rising tone. Tone
3 normally shows as a falling tone, but its movement is greatly influenced by tones before and after it. For example, when a tone 3 is preceded by a tone 3 or tone 4 and is followed by a tone 1 and tone 4 , its falling range is much smaller than in other cases. When a tone 3 is preceded by a tone 1 , it tends to move downward all the way. In other cases, its final portion tends to rise up. Tone 4 shows two patterns as well. When it is followed by a tone 1 or preceded by tone 3 or tone 4 , it shows as a high tone but with the final portion heads downward. In other cases, its final portion keeps flat or moves up.

Some of our observations have been documented in [7] but many are not. These phenomena are overlooked in traditional studies due to the lack of efficient ways to generate the statistics of tones in different context. There are many other phenomena can be seen from the CDTMs. Due to the limitation of space, we will not list all. But we want to point out that such context-dependent tone models contain much information, which are very useful data sources for studying the tonal system of Chinese dialects.

\section{DISCUSSION}

In this paper, we propose a statistical paradigm for prosody study. First, context dependent models are trained automatically from a natural speech corpus. Then data mining and visualization techniques are used to discover phonetic knowledge from the model parameters. By studying statistical features of CDTMs trained from a Xi' an dialect speech corpus, we learn much knowledge about the tonal system of the dialect. Some of our observations coincide with those from other studies, which validates the correctness of our approach. We also have many findings that haven't been reported, which demonstrates the advantages of our approach. We believe that such a paradigm is very helpful in reducing the data processing overhead for prosody study, especially for Chinese dialect study.

For the next step, we will apply this approach to other dialects and we will involve more contextual factors in model training. In this work, single speaker data is used. Some of our observations are common characteristics of the Xi' an dialect, but some may be speaker-related characteristics. We can verify them through investigating a multi-speaker speech corpus. Cross-dialect comparison is also in our plan.

\section{REFERENCES}

[1] K. Tokuda, T. Masuko, N. Miyazaki, and T. Kobayashi, "Multi-Space Probability Distribution HMM," IEICE Transactions on Information and Systems, vol. 85, no. 3, pp. 455-464, 2002.

[2] Hu Y., Chu M., and Huang C., "Exploring Tonal Variations via ContextDependent Tone Models," in Proc.Interspeech. ISCA, 2007.

[3] H. Wang, Y. Qian, F.K. Soong, J.L. Zhou, and J. Han, "A MultiSpace Distribution (MSD) Approach to Speech Recognition of Tonal Languages," in Ninth International Conference on Spoken Language Processing. ISCA, 2006.

[4] L. Breiman, Classification and Regression Trees, Chapman \& Hall/CRC, 1998.

[5] K. Shinoda and T. Watanabe, "MDL-based Context-dependent Subword Modeling for Speech Recognition," Acoustical Science and Technology, vol. 21, no. 2, pp. 79-86, 2000.

[6] J. Zhou, Y. Tian, Y. Shi, C. Huang, and E. Chang, "Tone Articulation Modeling for Mandarin Spontaneous Speech Recognition," IEEE International Conference on Acoustics, Speech, and Signal Processing, 2004

[7] R. Li, Dictionary of Xi'an Dialect, Jiangsu Educational Press, 1998. 


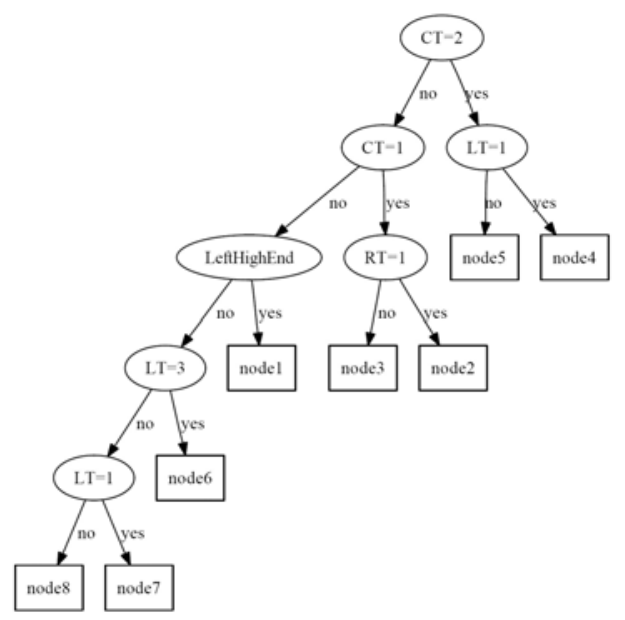

Fig. 3. The upper few layers of a decision tree for tying the second states of all context dependent tone models. On each node, question concerning certain contextual factor is asked to separate states into two groups according to their answers.

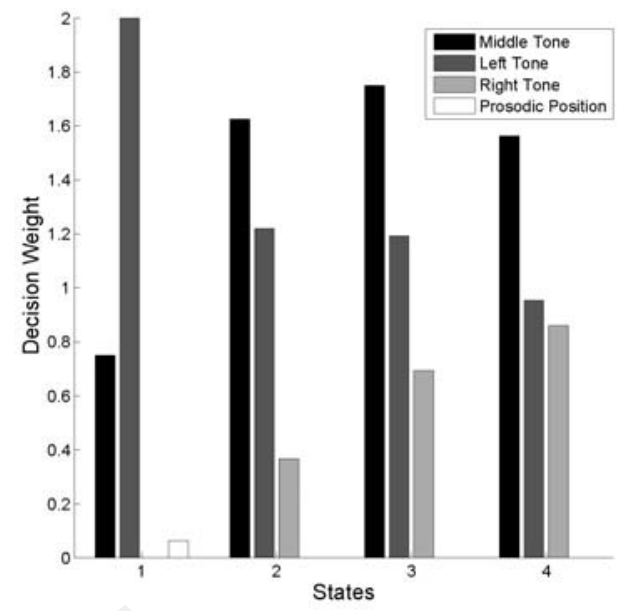

Fig. 4. The impacts of contextual factors on decision trees of different states. The decision weights indicates the how much certain kind of features are considered in node splitting.
$\mathrm{FO}(\mathrm{Hz})$
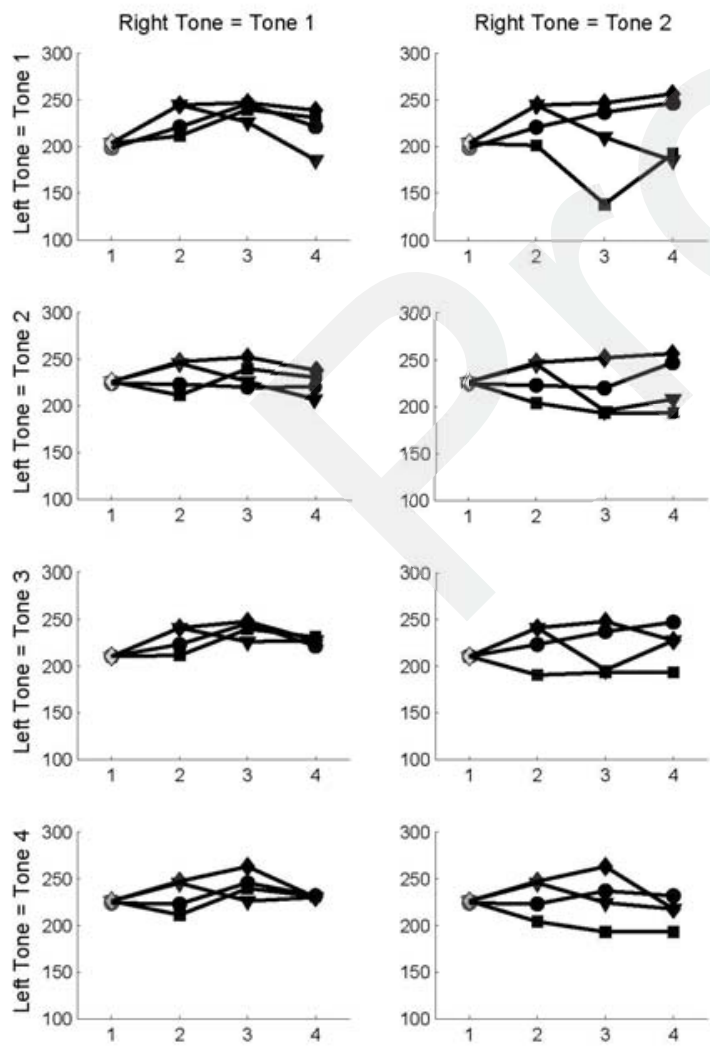
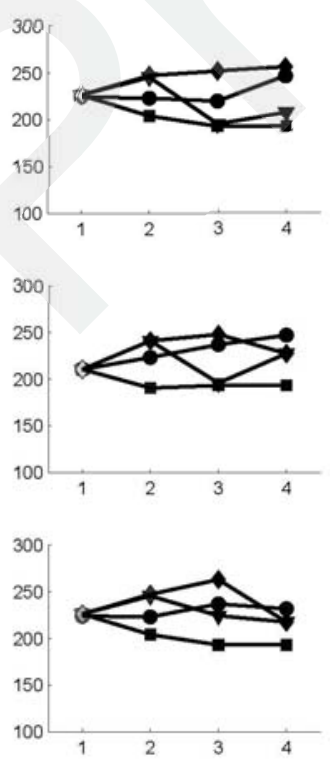

Right Tone $=$ Tone 3
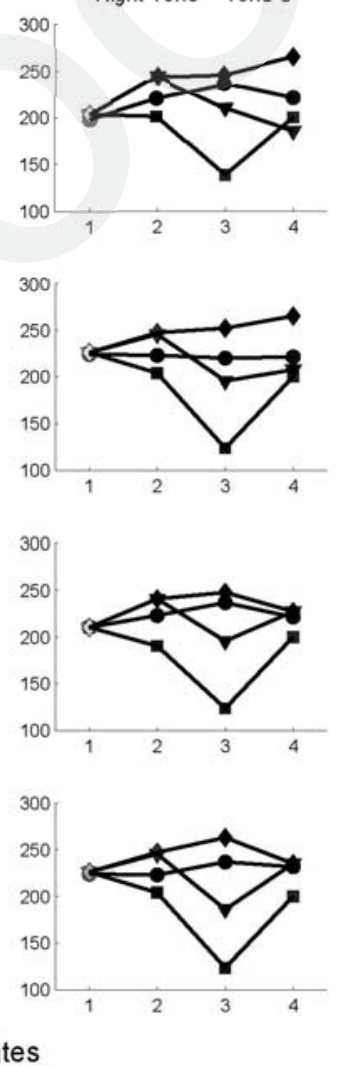

Right Tone $=$ Tone 4
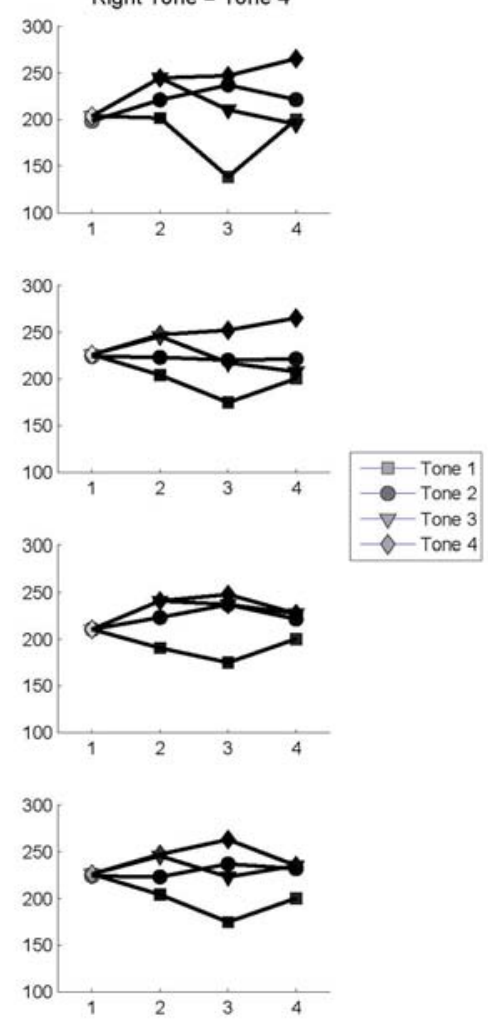

Fig. 5. The pitch curves of all tritone models for Xi'an dialect. In every subplot, 4 curves represent pitch curves of the syllables in 4 Mandarin tones respectively. Subplots within the same row are models with the same left tones, and subplots within the same column are models with the same right tones. Gray scale of the markers indicate their possibility of been voiced: the darker the higher the possibility. 